\title{
Aproximación multiproxy a los estudios paleoambientales de la provincia de Córdoba: el Valle de Ongamira como caso
}

\author{
Multiproxy approach for paleoenvironmental studies in \\ Córdoba Province. Ongamira valley as a case
}

VI Jornadas
Arqueológicas Cuyanas

Andrés D. Izeta*, G. Roxana Cattáneo**,
Andrés I. Robledo**, Julián Mignino****

*Instituto de Antropología de Córdoba (IDACOR), CONICET-Universidad Nacional de Córdoba y Museo de Antropología, Facultad de Filosofía y Humanidades, Universidad Nacional de Córdoba, Avenida Hipólito Yrigoyen 174, 5000, Córdoba, Argentina. E-mail: andresizeta@gmail.com

**Instituto de Antropología de Córdoba (IDACOR), CONICET-Universidad Nacional de Córdoba y Museo de Antropología, Facultad de Filosofía y Humanidades, Universidad Nacional de Córdoba, Avenida Hipólito Yrigoyen 174, 5000, Córdoba, Argentina. E-mail: roxanacattaneo@gmail.com

***Instituto de Antropología de Córdoba (IDACOR), CONICET-Universidad Nacional de Córdoba y Museo de Antropología, Facultad de Filosofía y Humanidades, Universidad Nacional de Córdoba, Avenida Hipólito Yrigoyen 174, 5000, Córdoba, Argentina. E-mail: and.robledo@gmail.com ****Museo de Antropología, Facultad de Filosofía y Humanidades, Universidad Nacional de Córdoba, Avenida Hipólito Yrigoyen 174, 5000, Córdoba, Argentina. E-mail: julianmignino@gmail.com

\begin{abstract}
Resumen
El objetivo general de este trabajo es el de contribuir con nueva información arqueológica que aporte a un modelo paleoecológico general para las Sierras Pampeanas Australes desde un caso de estudio: el valle de Ongamira, donde han sido planteados cambios, discontinuidades y procesos de complejización durante el Holoceno. Se pretende asociar estos procesos a un marco paleoambiental con datos multi-proxy (dataciones absolutas por ${ }^{14} \mathrm{C}$ y datos isotópicos asociados a estudios sedimentológicos, moluscos, microvertebrados y macro y micro-restos vegetales, entre otros).
\end{abstract}

Palabras clave: Córdoba; Arqueología; Holoceno; Cambios ambientales.

\begin{abstract}
The aim of this work is to contribute with new archaeological information to the human paleoecology model for the Southern Pampean Hills from a case study: the valley of Ongamira, where changes, discontinuities and complexity processes arise throughout the Holocene. It is intended to associate these processes to a multi-proxy data generated paleoenvironmental framework using absolute dating by ${ }^{14} \mathrm{C}$ and isotopic data associated with sedimentological studies, terrestrial gastropods shells, microvertebrates and macro and micro-debris from plants, among others.
\end{abstract}

Keywords: Córdoba; Archaeology; Holocene; Environmental changes.

El objetivo del presente trabajo es el de hacer una revisión sumaria sobre los datos relacionados con cambios paleoambientales y la ocupación humana de un espacio geográfico particular: un caso de estudio en las Sierras Pampeanas Australes de Argentina. Se propone avanzar en esta consideración mediante distintos tipos de datos. En primer lugar un análisis de los fechados radiocarbónicos de los sitios arqueológicos en la provincia de Córdoba, su desarrollo y uso durante los últimos 48 años. En segundo término presentar los modelos paleoambientales disponibles propuestos para las Sierras Pampeanas Australes y su relación con áreas vecinas. Por último proponemos discutir la utilización de estudios multi-proxy, con lo que se espera comprender de una manera más detallada los procesos que pueden haber afectado de distintos modos a las poblaciones e individuos que transitaron el actual territorio de las Sierras Pampeanas Australes y, más acotadamente, la zona de nuestro interés, el valle de Ongamira.

Desde el inicio de la arqueología argentina la región de las Sierras Pampeanas Australes ha sido tema de análisis 
por parte de distintos investigadores, en particular centrándose en el estudio de la evidencia arqueológica dejada por los grupos humanos que ocuparon el área que abarca el actual territorio de la provincia de Córdoba (e.g. Ameghino 1885; González 1960; Menghin y González 1954; Outes y Bruch 1910; Serrano 1945). En los últimos años, las evidencias pusieron de manifiesto una variabilidad en la manera en que estas poblaciones desarrollaron sus estrategias, adaptaciones y modos de vida a lo largo del tiempo desde los primeros asentamientos hace alrededor de 11.000 años, hasta los momentos previos a la conquista española (e.g. Berberián et al. 2011; Cattáneo et al. 2013; Laguens y Bonnin 2009; Rivero 2012). Para una síntesis de referencias bibliográficas puede verse Cattáneo et al. (2015: 291386); también allí puede encontrarse un relevamiento de gran parte de la información édita sobre la temática arqueológica de la provincia de Córdoba a la que se sumó información de análisis de colecciones de museos de los cuales se tomó registro de sus metadatos de procedencia, y en casos particulares, se realizaron prospecciones en el campo. Allí se compilaron los datos de un total de 1936 sitios arqueológicos registrados.

Esta base de datos se construyó sobre los productos de la investigación arqueológica efectuada por una gran diversidad de arqueólogos en las Sierras Pampeanas Australes a partir del año 1885 y hasta el año 2013. Esto trajo aparejado una gran variabilidad en cuanto a la calidad de los registros tanto publicados como no publicados. En muchos casos la falta de datos o las descripciones muy generales del sitio no permitieron establecer su localización exacta, sus cronologías o al menos determinar a partir de la cultura material asociada si pertenecen a ocupaciones de cazadores-recolectores generalizados, complejos o asentamientos agrícolas. Sin embargo, este compendio nos habilita a avanzar en un análisis de los datos de los sitios arqueológicos de la región en relación a su cronología estimada a los fines de comparar y discutir la distribución temporal encontrada y las posibles causas de la densidad de hallazgos a través del tiempo.

\section{Sitios arqueológicos y cronología}

Con el propósito del presente trabajo sólo hemos seleccionado los sitios arqueológicos que poseen edades radiocarbónicas a los fines de anclar desde una visión cronológica absoluta los cambios o continuidades y su relación con cambios paleoambientales. En relación a esto y con el fin de permitir la comparación regional o continental del registro arqueológico, hemos seguido el modelo de Walker et al. (2012) de la subdivisión del Holoceno a partir de los cambios paleoambientales y culturales registrados en diversos lugares del mundo. Los autores proponen una límite Pleistoceno- Holoceno Temprano fijado en 11700 cal AP, un Límite Holoceno Temprano-Medio en 8200 cal AP y un límite del Holoceno
Medio-Tardío a los 4200 cal AP, vinculados cada uno a una Sección y Punto del Estratotipo Global (GSSP).

La base de datos de la cronología de las Sierras Pampeanas Australes basada en fechados radiocarbónicos se desarrolló a partir de un total de 115 fechas correspondiente a 63 sitios (Cattáneo et al. 2015). Esta técnica de datación comenzó a utilizarse a finales de los años sesenta; el primer fechado corresponde al sitio Los Molinos (Marcellino et al. 1967), asignado a una ocupación de grupos agroalfareros del Departamento de Calamuchita.

A partir de ese trabajo pudo observarse que durante un período de 32 años (1967-1999) solo se realizaron 11 fechados radiocarbónicos (una tasa de casi una datación cada tres años). Desde el año 2005 dos programas se han implementado: uno centrado en restos humanos (e.g. Fabra 2009) y la otra en la cronología del Alero Deodoro Roca (Cattáneo et al. 2013, Cattáneo et al. 2015). Entre estos dos programas con base en el IDACOR-Museo de Antropología se encuentran el $52 \%$ de las edades radiocarbónicas a nivel regional (2010-presente, 73 fechados radiocarbónicos).

A los fines de evaluar la distribución de la serie de fechados se utilizaron modelos de sumas de probabilidades (e.g. Marsh 2015; Williams 2012) que se crearon mediante el uso del programa OxCal 4.2.4 (Bronk Ramsey 2009). Esto se basa en la necesidad de poder observar el comportamiento de las edades con sus probabilidades estadísticas en relación con los cambios paleoambientales registrados para la región.

La Figura 1 muestra las fechas de radiocarbono disponibles para el periodo 11500-presente, representadas como la suma de probabilidades. De acuerdo a lo propuesto por Williams (2012), debemos ser cautos en las interpretaciones debido a que las distribuciones podrían estar ocurriendo por diferentes variables, como la falta de estudios arqueológicos, problemas geomorfológicos o tafonómicos. Sin embargo, y teniendo esto presente, pueden observarse ciertas características o distribuciones probabilísticas de densidades de fechados que poseen particularidades.

Por un lado, hay tres "vacíos" en la serie delimitados por la distribución calculada. Para el Holoceno Temprano los fechados se encuentran agrupados cerca del $11000 \mathrm{cal}$ AP y con uno cuya media se ubica cerca del 9700 cal AP. El siguiente grupo se coloca en el Holoceno Medio y el límite más antiguo de este conjunto aparece alrededor de 7100 cal AP. Aquí se hace evidente un primer vacío de más de 2400 años entre éste y las primeras fechas. Esta situación no es sorprendente, ya que ha sido planteado que las condiciones medioambientales del evento de los 8200 cal AP y la inestabilidad del Holoceno Medio se registran en localidades de todo el mundo, 


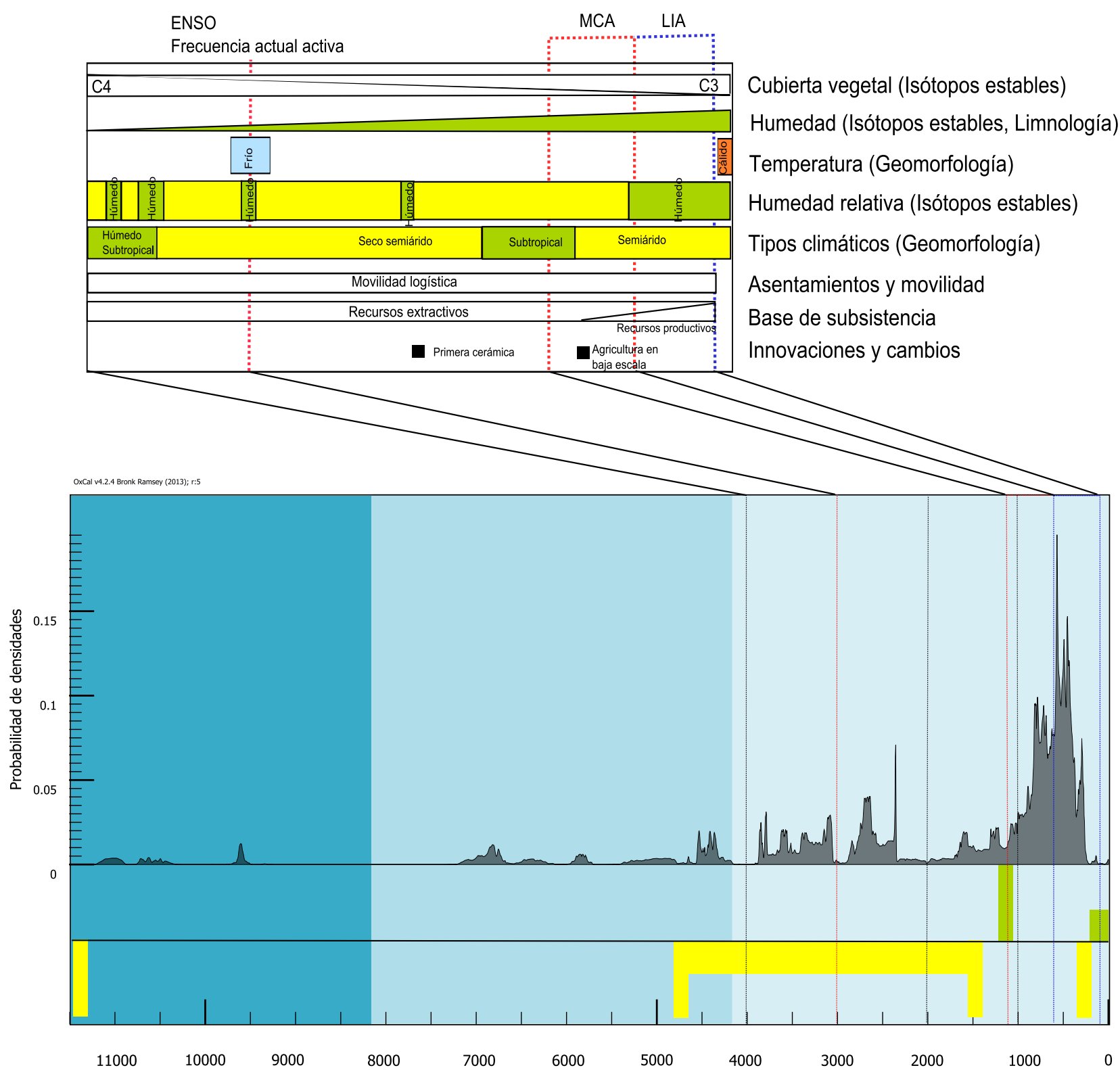

Figura 1. Suma de probabilidades de las fechas de radiocarbono disponibles para el periodo 11500-presente.

Figure 1. Radiocarbon dates sum of probabilities. From 11500 BP to the present.

incluyendo por supuesto a América del Sur (Walker et al. 2012). Esto hace del Holoceno Medio un período de eventos climáticos globales significativos en términos de sus efectos sobre diversas comunidades humanas. Generalmente, ha sido relacionado con el cambio cultural, la diversificación global o la variabilidad y, más localmente, con una probable disminución en la demografía de las ocupaciones humanas (e.g. Rivero 2012). De todos modos esto último debe verificarse dado que la escasa cantidad de sitios arqueológicos para este momento puede deberse al poco número de investigadores centrados en el estudio de estas sociedades, la evaluación de otros probables procesos que estén generando sesgos en el registro y la necesidad de interpretar de un modo más específico las condiciones ambientales locales (Figura 1).
Un segundo vacío de fechados se observa en torno a los 6500 y 5500 años AP, justo en el punto medio del Holoceno Medio cuando, de acuerdo a Giorgis et al. (2015), las condiciones locales habrían girado hacia un clima un poco más cálido y húmedo.

El tercero se sitúa alrededor del límite de 4200 cal AP. Esto coincide con un aumento de la aridez, como se refleja en el registro paleoambiental local y el de América del Sur (e.g. Giorgis et al. 2015, Piovano et al. 2009). A modo de ejemplo, las condiciones de sequía se indican con un marcado incremento en el contenido de polvo en el núcleo de hielo del Nevado Huascarán del norte de Perú, y son evidentes en datos de diatomeas y sedimentos del Lago Titicaca (Walker et al. 2012). A nivel local, el 
análisis isotópico de paleosuelos, los niveles de agua de la laguna de Mar Chiquita y el de las escorrentías de aguas superficiales indican la misma tendencia (e.g. Piovano et al. 2009, Silva et al. 2011).

El porqué de estas tendencias generales a nivel espacial se relaciona con la distribución del clima sudamericano, que se encuentra estrechamente vinculado a la particular forma cónica y la topografía del continente, combinada con la ubicación austral y la altura de los Andes, las celdas de circulación global, las corrientes oceánicas y la proximidad de grandes masas de agua. Entre los principales componentes de los patrones de circulación atmosférica estacionales de América del Sur están el Monzón de verano Sudamericano y las corrientes en chorro de bajo nivel Sudamericanos (Piovano et al. 2009). Otra característica del sur de Sudamérica está proporcionada por el desarrollo de un área continua con baja pluviosidad (de 250 milímetros por año) que cruza el continente en dirección noroeste-sudeste desde Perú a la región costera de la Patagonia argentina, llamada "Diagonal Árida". La región norte y este de la Diagonal Árida (por ejemplo, el centro de Argentina) reciben la humedad de origen Atlántico con precipitación máxima durante el verano austral. Por el contrario, la región occidental de la Diagonal Árida (Patagonia) se encuentra bajo una influencia pacífica y se caracteriza por la precipitación máxima durante el invierno. En este sentido, el análisis de los registros climáticos en el norte y este de la Diagonal árida ha permitido reconstruir la actividad pasada del Sistema Monzónico de verano de Sur América (e.g. Bruniard 1982). Es conocido para el Holoceno que las regiones al norte de la Diagonal se vieron afectados por intervalos largos de sequía seguidos de un período húmedo vinculado a las tendencias a largo plazo y las teleconexiones interanuales de El NiñoOscilación del Sur (ENSO) (Piovano et al. 2009). Entender el comportamiento, y particularmente el corrimiento, de esta Diagonal árida a través del tiempo es fundamental para comprender el pasado de las poblaciones humanas dada su relación con las comunidades vegetales y animales quienes dependen fuertemente del clima por lo que creemos que todo dato que aporte a describir esta movilidad en términos de cronología absoluta es indispensable.

\section{La información paleoambiental regional disponible}

El registro paleoambiental más completo del Holoceno se ha llevado a cabo en la Laguna Mar Chiquita, un enorme cuerpo de agua salina ubicado a $150 \mathrm{~km}$ al este de Ongamira. Allí, Piovano y colegas (2009) caracterizan variaciones paleohidrológicas a través del Holoceno, notando que la fase húmeda y cálida del Holoceno Temprano y Medio se vio interrumpida por un cambio hidrológico que muestra una fase super-seca con una magnitud extrema alrededor de 4700 cal AP. La aridez general fue dominante hasta mediados del primer milenio $A D$, cuando se pueden deducir bajantes menos extremas. Las condiciones secas durante el Holoceno Medio han sido ampliamente reconocidas para las Ilanuras pampeanas subtropicales, apuntando hacia una magnitud regional del evento climático frío y seco. La fase fría y seca del Holoceno Medio es consistente con un reducido transporte latitudinal de la humedad desde los trópicos hasta las regiones subtropicales, como consecuencia de una circulación monzónica debilitada (Piovano et al. 2009).

En relación a esto, estudios actuales de la vegetación del Bosque Chaqueño Serrano (Cabido et al. 2008; Silva et al. 2011, Giorgis et al. 2015) desde distintas perspectivas permiten discutir la variabilidad en los cambios de las composiciones florísticas en distintas zonas ecológicas a través del tiempo (Cagnolo et al. 2006; Cabido et al. 2008; Giorgis et al. 2011, 2015, entre otros). En este sentido, Silva et al. (2011) plantean para el Holoceno Tardío cambios de frecuencias de taxones en base a estudios de isótopos estables del carbono en suelos. En concreto postulan que diversos ecosistemas dominados por plantas del tipo $\mathrm{C}_{4}$ (gramíneas) fueron cambiando desde el 3800 cal AP hacia otros mayormente compuestos por taxones florísticos del tipo $C_{3}$ (herbáceas y leñosas). Teniendo en cuenta que las plantas $C_{4}$ se ajustan a condiciones ambientales más secas y cálidas que las del tipo $C_{3}$, puede interpretarse que este cambio en la composición florística puede relacionarse a los cambios ambientales registrados por otros proxys para el Holoceno Tardío Local.

Por otro lado, Giorgis et al. (2015) postulan el mismo tipo de cambio de las condiciones ambientales desde el análisis de silicofitolitos recuperados de las mismas localidades muestreadas por Silva et al. (2011) y del sitio arqueológico El Alto 3, lugares en su mayoría ubicados a una distancia variable entre los 100 a $200 \mathrm{~km}$ de nuestra área de estudio. A partir de estos análisis los autores caracterizan la evolución climática, la cual en términos generales responde a los modelos regionales, aunque presenta algunas variaciones que pueden asociarse a las condiciones locales donde se ubica el sitio arqueológico.

Por otro lado, históricamente se puede observar que los estudios paleoambientales para la región central de la Argentina han sido realizados a lo largo del tiempo y en gran medida en base a interpretaciones geológicas. Ameghino (1885) y Doering (1882) ya habían descripto secuencias para el Holoceno determinando la presencia de diversas depositaciones sedimentarias cuaternarias. Otros investigadores como Castellanos (1933) y Montes (1950), este último asesorado por el geólogo Olsacher, también realizaron minuciosos estudios sobre los paleoambientes del Holoceno relacionados particularmente con ocupaciones humanas. Sin embargo es a partir de la década de 1980 que comienza el trabajo sistemático en el área que resulta en la implementación de 
proyectos interdisciplinarios desde una mirada ecológica. Específicamente es el valle de Copacabana el que recibe atención y que produce conocimiento científico actualizado (e.g. Bonnin et al. 1985, Cattáneo et al. 1994, Laguens 1999, Laguens et al. 1987). Las caracterizaciones geológicas continuaron (Carignano 1999), donde se interpretan las variaciones ambientales en base a las fluctuaciones climáticas entre el Pleistoceno y el Holoceno mediante estudios geomorfológicos. Cioccale (1999) también planteó un modelo paleoambiental basado en una aproximación multivariada (geológico, climatológico, histórico y arqueológico) para el último milenio $A D$. Bonnin et al. (1985), como se dijo más arriba, también caracterizaron los cambios ambientales ocurridos en el Valle de Copacabana, en el norte de las Sierras Chicas cordobesas desde una mirada paleoecológica. Soibelzon et al. (2013), entre otros, realizaron análisis paleoambientales en base a la caracterización de la fauna de algunos sitios arqueológicos del tramo final del Holoceno tardío de las Sierras Pampeanas Australes. Por ejemplo, para estos autores, la mayor frecuencia de armadillos a partir del 900 AD estaría asociado a un aumento de temperatura y precipitaciones.

\section{El caso del Alero Deodoro Roca en el Valle de Ongamira}

Para centrar el análisis de caso en este marco presentaremos la localidad del valle de Ongamira ubicado en las Sierras Pampeanas Australes en el extremo norte de las Sierras Chicas, una cadena montañosa mediterránea a más de 1000 km de las costas del Atlántico Sur y del Pacífico. Allí desde 2010 comenzó a ser estudiado un sitio arqueológico multicomponente, el Alero Deodoro Roca (ADR), del que fue posible recuperar diversos tipos de muestras que nos permiten aproximarnos a interpretaciones paleoambientales del último tramo del Holoceno. Nos referiremos a estudios isotópicos de valvas de moluscos terrestres, de microvertebrados y de restos antracológicos de plantas leñosas.

\section{Análisis isotópicos}

Dado que es uno de los pocos sitios arqueológicos en el centro de Argentina que contiene acumulaciones de conchas terrestres asociadas con ocupaciones de sociedades cazadoras-recolectoras prehispánicas, se focalizó el interés en análisis químicos, pues la composición isotópica de gasterópodos terrestres se está utilizando cada vez más como un proxy paleoambiental (Yanes et al. 2014). En este sentido, se analizaron restos de la especie Plagiodontes daedaleus recuperados del Sector B de ADR. También se recogieron muestras modernas de valvas, agua de lluvia, suelo y vegetación actual para adquirir la señal isotópica moderna del valle y el sitio. Este primer paso nos permitió notar que las muestras arqueológicas no estaban contaminadas con valores isotópicos modernos, los que fueron significativamente diferentes. Luego observamos que las valvas arqueológicas enteras mostraron valores que eran, en promedio, alrededor de $2.5 \%$ más altos en el $\delta^{13} \mathrm{C}$ y alrededor de $1.8 \%$ más altos en el $\delta^{18} \mathrm{O}$ que en los especímenes modernos, incluso si se realizaba la corrección por el efecto Suess (Yanes et al. 2014: 1198). Puede interpretarse a través de los resultados obtenidos a partir del $\delta^{18} \mathrm{O}$ que hubo una menor humedad relativa y/o una mayor cantidad de lluvias y que las plantas $C_{4}$ fueron más abundantes hacia comienzos del Holoceno Tardío, que en los tiempos actuales. Aquí vale la pena remarcar que en las unidades estratigráficas fechadas antes del límite de 4200 cal AP no hay presencia de caracoles terrestres, que necesitan una humedad relativa alta; en cambio para las unidades post 4200 cal AP la presencia de gasterópodos es masiva (Izeta et al. 2014). Estos hallazgos no coinciden plenamente con los otros datos de la región ya mencionados previamente (e.g. Silva et al. 2011) sugiriendo, entonces, que el clima micro local del Holoceno Tardío posee variaciones en relación al del Holoceno Medio para áreas circundantes. Esto mismo se ve sustentado en la composición faunística diferencial (Costa 2015) y la composición sedimentológica distinta entre las unidades estratigráficas de ADR (Zárate 2016).

Esto es algo que también se observa en el registro del sitio El Alto 3 en donde se reconoce una variabilidad en cuanto al ajuste de fechas a los modelos ambientales regionales como fuera descripto en Giorgis et al. (2015).

\section{Estudios de microverterbrados}

Para sumar información ambiental local se trabajó sobre la base de la identificación de los taxones representados en ensambles de micromamíferos recuperados en excavaciones y de muestreos actualísticos de egagrópilas de aves rapaces (Mignino et al. 2014, 2016).

Por un lado, se estudió un conjunto arqueológico (NSP $=473)$ de ADR compuesto por restos cráneomandibulares y post-craneales asociados a fechados de ca. 3000 cal AP (Cattáneo et al. 2013).

Conjuntamente, se analizó una muestra actualística obtenida a partir de la recolección sistemática y estacional de egagrópilas de aves rapaces que habitan en el mismo sitio. Para este caso se estudiaron un total de 26 bolos, los cuales permitieron identificar un total de 83 individuos entre los cuales se incluyen insectos, aves, roedores y marsupiales.

Siguiendo la propuesta metodológica de Andrews (1990) y Fernández-Jalvo y Andrews (1992), todos los conjuntos fueron sometidos a exámenes tafonómicos para entender las diferencias entre las concentraciones naturales y las acumulaciones óseas culturales en el sitio. Esto permitió interpretar a los conjuntos recuperados de contextos arqueológicos como parte de bolos de regurgitación 


\begin{tabular}{lcccc}
\hline Taxones/Procedencia & \multicolumn{2}{c}{ ADR } & \multicolumn{2}{c}{ ADR } \\
& NSP & MNI & NSP & MNI \\
\hline AVES & 4 & 2 & 1 & 1 \\
Mammalia/Rodentia & 4 & 2 & 7 & 4 \\
Ctenomyidae & 1 & - & - & - \\
Ctenomys sp. & 2 & 1 & 21 & 14 \\
Caviidae & - & - & - & - \\
Microcavia australis & 5 & 3 & 25 & 9 \\
Cricetidae/Sigmodontinae & - & - & - & - \\
Phyllotis xanthopygus & 91 & 34 & 4 & 3 \\
Reithrodon auritus & - & - & 12 & 6 \\
Calomys musculinus & 47 & 23 & - & - \\
Akodon polopi & 15 & 6 & - & - \\
Akodon sp. & - & - & 4 & 3 \\
Didelphidae & - & - & - & - \\
Thylamys pallidior & 25 & 12 & - & - \\
Total & 192 & 83 & 74 & 40 \\
\hline
\end{tabular}

Tabla 1. Composición taxonómica de conjuntos actuales y arqueológicos de micro vertebrados de ADR.

Table 1. Taxonomic composition of modern and archaeological micro vertebrate assemblages from ADR site.

diversos patrones culturales utilizadas para la selección de la leña en las región semiárida de Córdoba ya descriptas por Martínez (2015). Sin embargo, para el período más tardío de estudio, ca. 1900 años AP, existe un aumento en los restos carbonizados de Celtis sp., Condalia sp., Prosopis sp., Geoffraea sp. y Ziziphus sp. Su presencia podría indicar una correspondencia con un clima más propicio a condiciones húmedas y de desarrollo en suelos característicos de zonas serranas de acuerdo a sus características ecológicas (Zuloaga et al. 2008; Demaio et al. 2002).

antiguos (Mignino et al. 2014). Como resultado, si bien los ensambles específicos entre ambos conjuntos no difieren significativamente, la principal diferencia radica en el hecho de que en la muestra arqueológica se registró la presencia de abundantes restos de un roedor sigmodontino característico de ambientes más fríos y secos (Reithrodon auritus). Esta especie no se ha registrado en las muestras modernas y su nicho parece estar ocupado por otra especie de roedor de hábitos más generalistas, también sigmodontino (Calomys musculinus) y una especie de pequeño marsupial sudamericano (Thyllamys pallidior) (Tabla 1). Aunque necesitamos, a efectos comparativos, un número mayor de muestras a escala regional, la diferencia preliminar en abundancia y composición de taxones entre los dos conjuntos en ADR podría estar relacionada con un cambio ambiental de un clima frío y seco a uno más cálido y húmedo. En general, esto coincide con la información registrada a partir de otros proxy ambientales regionales.

\section{Estudios antracológicos}

Desde el año 2014 se realizó un estudio tafonómicamente orientado de más de 5000 fragmentos de carbón provenientes de las excavaciones de ADR (Robledo 2016a, 2016b). De esta muestra, más de un centenar fueron identificados, al menos, a nivel de género. Algunas diferencias fueron advertidas y en este sentido hemos utilizado los estudios antracológicos de Ongamira como otro proxy paleoambiental.

La alta variabilidad presente en el registro de leñosas (18 taxones), diferenciada en cuatro momentos temporales (ca. 1900, ca. 3000, ca. 3600 y ca. 4000 AP, Tabla 2), puede tener múltiples interpretaciones debido a los procesos culturales que operan sobre las especies vegetales y sus usos (Robledo 2016a, 2016b: 172), particularmente la incorporación selectiva de las leñosas con frutos comestibles (Arenas 2003) o los
Para el componente ca. 3000 años AP se describieron una mayor variedad de especies (14 taxones) siendo más representativos los de Acacia sp., Lithraea sp., Ruprechtia sp. y Cercidium sp., este último asociado a la idea de otros usos además de la combustión a partir de la obtención de su resina (brea) (Robledo 2016b:182). Por otra parte, la composición taxonómica de estos dos componentes (ca. 1900 y ca. 3000 años AP) es la que presenta mayor variabilidad de taxones, no así el componente de ca. 3600 años AP que tiene la mitad de especies representadas (Robledo 2016b:169). Finalmente para el caso del componente más temprano (ca. 4000 AP) solo se registró el taxón Castela sp. Si bien la muestra es de menor tamaño, debido a que la superficie excavada es menor, las características nos sugieren un aprovisionamiento de leña ya seca debido a la presencia de huecos de xilófagos en los restos antracológicos (Robledo 2016b:180).

Sin dudas, la presencia de estos taxones no es determinante de un clima específico dado que está mediada por distintos filtros naturales y culturales que afectan su composición (Théry-Parisot et al. 2010), entre ellos, la combustión diferencial (ver discusión en Robledo 2016b:172). No obstante, la mayor presencia de diversidad taxonómica en los conjuntos más tardíos y la presencia de restos de leñosas son consistentes con una mayor disponibilidad de plantas $C_{3^{\prime}}$ lo que es coincidente con los resultados obtenidos por otros análisis (Silva et al. 2011). Si bien no existen otros trabajos con los cuales comparar estos resultados, los datos provisorios aportados ayudan a integrar la antracología de ADR como un nuevo proxy para los estudios paleoambientales locales.

\section{Discusión y Conclusiones}

El registro arqueológico de la provincia de Córdoba con dataciones absolutas presenta un desbalance entre aquellos sitios con cronologías tempranas (Holoceno 


\begin{tabular}{|c|c|c|c|c|c|c|}
\hline & Taxón & $\begin{array}{l}\text { Componente } \\
\text { Temporal ca. } \\
1900 \text { años AP }\end{array}$ & $\begin{array}{l}\text { Componente } \\
\text { Temporal ca. } \\
3000 \text { años AP }\end{array}$ & $\begin{array}{l}\text { Componente } \\
\text { Temporal ca. } \\
3600 \text { años AP }\end{array}$ & $\begin{array}{l}\text { Componente } \\
\text { Temporal ca. } \\
4000 \text { años AP }\end{array}$ & Totales \\
\hline \multirow{3}{*}{ Anacardiaceae } & Lithraea sp. & 16 & 10 & 3 & 0 & 29 \\
\hline & Schinopsis sp. & 3 & 5 & 4 & 0 & 12 \\
\hline & Schinus sp. & 1 & 0 & 0 & 0 & 1 \\
\hline Celtidaceae & Celtis sp. & 1 & 1 & 0 & 0 & 2 \\
\hline \multirow{5}{*}{ Fabaceae } & Acacia sp. & 1 & 19 & 3 & 0 & 23 \\
\hline & Cercidium sp. & 5 & 14 & 9 & 0 & 28 \\
\hline & Geoffraea sp. & 5 & 0 & 0 & 0 & 5 \\
\hline & Prosopis sp. & 1 & 0 & 1 & 0 & 2 \\
\hline & Senna sp. & 2 & 1 & 0 & 0 & 3 \\
\hline Nyctaginaceae & Bougainvillea sp. & 2 & 1 & 3 & 0 & 6 \\
\hline Polygonaceae & Ruprechtia sp. & 0 & 11 & 0 & 0 & 11 \\
\hline \multirow{2}{*}{ Rhamnaceae } & Condalia sp. & 4 & 4 & 1 & 0 & 9 \\
\hline & Ziziphus sp. & 1 & 0 & 0 & 0 & 1 \\
\hline Rosaceae & Polylepis sp. & 0 & 1 & 0 & 0 & 1 \\
\hline Rutaceae & Zanthoxylum sp. & 6 & 2 & 7 & 0 & 15 \\
\hline Santalaceae & Jodina sp. & 0 & 1 & 0 & 0 & 1 \\
\hline Simaroubaceae & Castela sp. & 1 & 3 & 0 & 3 & 7 \\
\hline \multirow[t]{2}{*}{ Zygophyllaceae } & Porliera sp. & 0 & 1 & 0 & 0 & 1 \\
\hline & Totales & 49 & 74 & 31 & 3 & 157 \\
\hline
\end{tabular}

Tabla 2. Listado de taxones identificados en el material arqueológico por Componente Temporal (plantas leñosas).

Table 2. List of identified taxa grouped in chronological components (woody plants).

Temprano y Medio) en relación con las del Holoceno Tardío. Incluso dentro de este último lapso la gran mayoría de las ocupaciones se agrupan hacia el tramo final del mismo (Figura 1).

Por otro lado, el registro paleoambiental se ha ido construyendo desde diversos campos disciplinares, logrando definir historias ambientales locales que se ajustan en términos generales a las tendencias globales. En este sentido, los cambios observados en los proxys elegidos apuntan a esbozar una tendencia a escala local de estas tendencias generales.

Los datos obtenidos a partir de estudios paleohidrológicos y paleolimnológicos para la región de Mar Chiquita (Piovano et al. 2009) presentan resultados similares a los que pudimos interpretar en ADR a partir de los resultados de estudios isotópicos en valvas de gasterópodos terrestres $\left(\delta^{18} \mathrm{O}\right)$. Esto denota un aumento de la humedad acaecida alrededor de los 3800 años AP, fecha en la cual también pudo observarse un cambio en las comunidades vegetales a nivel regional, tal como observaron Silva et al. (2010). Las diferencias en los elencos faunísticos y en los restos antracológicos, aunque un poco más débil, también apoyan este cambio en las condiciones ambientales locales y regionales.
Resumiendo, podemos concluir que los datos generados durante la investigación de ADR apoyan, en general, a otros proxy, que atribuyen una variabilidad paleoambiental durante el Holoceno tardío donde se hacen más evidentes los cambios de las condiciones ambientales que van desde instancias más cálidas, secas y áridas, hasta las más húmedas y frías.

Para el Holoceno Temprano y Medio, ADR aún no ha podido ofrecer información, ya que los fechados que ubican a algunas ocupaciones en este momento se han realizado en el Sector A (Cattáneo et al. 2013), cuya excavación no ha sido aún retomada. Si bien se conoce su antigüedad, no tenemos conocimiento de las asociaciones de cultura material para esa fecha. Por ello creemos que la evidencia es particularmente escasa para permitir hablar de procesos generales o de mayor escala (sólo existe registro de tres sitios para el Holoceno temprano y ocho sitios para el Holoceno Medio con fechados radiocarbónicos asociados). Por lo tanto, para indagar en momentos más antiguos se debe desarrollar un programa centrado en la localización y la datación de los sitios más tempranos en la estratigrafía de manera que enriquezcan el debate y la integran en un marco paleoambiental, a escala local y continental. En este escenario, el Alero Deodoro Roca recobra importancia 
debido a sus fechas del Holoceno Medio y los sedimentos pendientes de excavación. Finalmente, las asociaciones de datos presentadas nos permiten incluir un conjunto de información ambiental de interés para la comprensión de los procesos paleoclimáticos generales en los cuales se desarrollaron las poblaciones prehispánicas de la región que futuros estudios permitirán profundizar.

\section{Agradecimientos}

Este trabajo ha sido financiado con fondos de subsidios pertenecientes al PICT 2011-2122, PIP CONICET 2011 y 2014, y SECYT UNC 2010-2015. Los autores desean agradecer a los organizadores de las VI Jornadas Cuyanas por su invitación a publicar este trabajo.

\section{Bibliografía}

Andrews, P. 1990. Owls, caves and fossils. University of Chicago Press, Chicago.

Ameghino. F. 1885. Informe sobre el Museo Antropológico y Paleontológico de la Universidad Nacional de Córdoba durante el año 1885. Boletín de la Academia Nacional de Ciencias de Córdoba, VIII: 347-360.

Arenas, P. 2003. Etnografía y alimentación entre los toba-ñachilamoleek y wichi-lhuku'tas del Chaco Central (Argentina), Edición del autor, Buenos Aires.

Berberián E. E., Bixio, B., Bonofiglio, M., González, M.C., Medina, M.E., Pastor, S., Recalde, M.A., Rivero, D.E. \& Salazar, J. 2011. Los pueblos indígenas de Córdoba. Ediciones del Copista, Biblioteca de Historia, Córdoba.

Bonnin, M., Laguens, A. \& Díaz, S. 1985. Ambiente actual y pasado en la cuenca del Río Copacabana (Departamento Ischilín, Provincia de Córdoba). Publicaciones del Instituto de Antropología XLV: 159-201.

Bronk Ramsey, C. 2009. Bayesian analysis of radiocarbon dates. Radiocarbon 51, 337-60.

Bruniard, E. D. 1982. La diagonal árida argentina: un límite climático real. Revista Geográfica 95: 5-20

Cabido M, Pons E, Cantero J. J., Lewis J. P. \& Anton A., 2008. Photosynthetic pathway variation among C4 grasses along a precipitation gradient in Argentina. Journal of Biogeography 35: 131-140.

Cagnolo L., Cabido M. \& Valladares G. 2006. Plant species richness in the Chacho Serrano Woodland from Central Argentina: Ecological traits and habitat fragmentation effects. Biological conservation 132: 510.

Carignano, C. 1999. Late Pleistocene to recent climate change in Córdoba Province, Argentina: Geomorphological evidence. Quaternary International 57-58: 117-134
Castellanos, A. 1933. El hombre prehistórico de la Provincia de Córdoba. Revista de la Sociedad de Amigos de la Arqueología VII: 5-88.

Cattáneo, R., M. Bonnin \& A. Laguens. 1994. Adaptaciones humanas durante el Holoceno a ambientes de bosque chaquense en Argentina. Actas y Memorias del XI Congreso Nacional de Arqueología Argentina, Revista del Museo de Historia Natural de San Rafael XIV (1/4): 63-66.

Cattáneo G.R., A. D. Izeta \& M. Takigami. 2013. Primeros fechados radiocarbónicos para el Sector B del sitio Alero Deodoro Roca (Ongamira, Córdoba, Argentina). Relaciones de la Sociedad Argentina de Antropología 38 (2): 559-567.

Cattáneo, G.R., M. Zárate \& A. D. Izeta. 2013. Informe de sedimentos recuperados en Perfil Norte de la cuadricula $X B$ del Sector $B$ del sitio Alero Deodoro Roca. Ms.

Cattáneo, G. R., A. D. Izeta \& T. Costa. 2015. El patrimonio arqueológico de los espacios rurales de la provincia de Córdoba. Museo de Antropología-IDACOR: Córdoba.

Cioccale, M. 1999 Climatic fluctuations in the Central Region of Argentina in the last 1000 years. Quaternary International 62: 35-47.

Costa, T. 2015. Los Humanos, los animales y el territorio. Sus interacciones en el pasado en la Sierras Pampeanas Australes, provincia de Córdoba, Argentina. Tesis doctoral inédita. Facultad de Filosofía y Humanidades, Universidad Nacional de Córdoba.

Demaio, P., Karlin, U.O. \& Medina, M. 2002. Árboles nativos del centro de Argentina. LOLA Botánica (Eds): Buenos Aires.

Doering, A. 1882. Geología. Informe oficial de la Comisión Científica agregada al estado mayor general de la expedición al Río Negro (Patagonia) III: 1-128.

Fabra, M. 2009. El poblamiento prehispánico de Córdoba: una interpretación a partir de evidencias bioantropológicas. Serie Tesis de Postgrado. Córdoba, Centro de Publicaciones Facultad de Filosofía y Humanidades, Universidad Nacional de Córdoba.

Fernández-Jalvo Y. \& P. Andrews. 1992. Small Mammal Taphonomy of Gran Dolina, Atapuerca (Burgos), Spain. Journal of Archaeological Science 19: 407-428.

Giorgis, M. A., A. M. Cingolani, F. Chiarini, J. Chiapella, G. Barboza, L. Ariza Espinar, R. Morero, D. E. Gurvich, P.A. Tecco, R. Subils \& M. Cabido. 2011. Composición florística del Bosque Chaqueño Serrano en la provincia 
de Córdoba, Argentina. Kurtziana 36 (1): 9-43.

Giorgis, M A. M. L. López, D. Rivero \& A. M. Cingolani. 2015. Cambios climáticos en las sierras de Córdoba (Argentina) durante el holoceno. Aportes a las reconstrucciones climáticas a través del análisis de silicofitolitos del sitio arqueológico El Alto 3. Boletín de la Sociedad Argentina de Botánica, 50 (3): 361-375.

González, A R. 1960. La estratigrafía de la gruta de Intihuasi (Prov. de San Luis, R.A.) y sus relaciones con otros sitios precerámicos de Sudamérica. Revista del Instituto de Antropología I: 1-290. Universidad Nacional de Córdoba.

Izeta, A. D., Costa, T., Gordillo, S., Cattáneo, R., Boretto, G., Robledo, A. 2014. Los gasterópodos del sitio Deodoro Roca, Valle de Ongamira (Córdoba, Argentina). Un análisis preliminar. Revista Chilena de Antropología 29: 74-80

Laguens A. G. 1999. Arqueología del contacto hispano indígena. Un estudio de cambios y continuidades en las Sierras Centrales de Argentina. BAR, International Series 801. Oxford.

Laguens, A.G. \& M.I. Bonnin. 2009. Sociedades Indígenas de las Sierras Centrales. Arqueología de Córdoba y San Luis. Editorial Universitaria, Universidad Nacional de Córdoba.

Laguens, A.G., M.I Bonnin\& S. Díaz. 1987. Programa Chuña. Publicaciones del Instituto de Antropología (Córdoba) 45: 9-14.

Marcellino, A., E. Berberián \& J. Pérez 1967 El yacimiento arqueológico de Los Molinos (Dpto. Calamuchita Córdoba). Publicaciones del Instituto de Antropología XXVI: 1-68.

Marsh, E. J. 2015. The emergence of agropastoralism: Accelerated ecocultural change on the Andean altiplano, 3540-3120 cal BP. Environmental Archaeology 20 (1): 13-29.

Martínez, G. J. 2015. Cultural patterns of firewood use as a tool for conservation: A study of multiple perceptions in a semiarid region of Cordoba, Central Argentina. Journal of Arid Environments 121: 84-99.

Menghin, O. A. F. \& A. R. González 1954 Excavaciones arqueológicas en el yacimiento de Ongamira, Córdoba (Rep. Arg.) (Nota preliminar). Notas del Museo de La Plata XVII, Antropología 67: 213-274.

Mignino, J., J. J. Martínez \& A.D. Izeta. 2014. Late Holocene ( 3.9 kybp-present) environmental conditions through the analysis of microfauna. Upper Ongamira Valley, Northern Córdoba Province, Central Argentina.
XII International Conference International Council for Archaeozoology, San Rafael, 22-27 Septiembre 2014

Montes, A. 1950. Nomenclador Cordobense de toponimia autóctona (primera parte). Anales de Arqueología y Etnología XI: 33-80.

Outes, F. \& C. Bruch. 1910. Los aborígenes argentinos. Estrada, Buenos Aires.

Piovano E. L., D. Ariztegui, F.Cordoba, M. Cioccale \& F. Sylvestre. 2009. Hydrological Variability in South America Below the Tropic of Capricorn ( Pampas and Patagonia, Argentina) During the Last $13.0 \mathrm{Ka}, \mathrm{F}$. Vimeux et al. ( eds. ), Past Climate Variability in South America and Surrounding Regions, Developments in Paleoenvironmental Research 14: 323-351.

Rivero, D. 2012. La ocupación humana durante la transición Pleistoceno-Holoceno (11,000 - 9000 A.P.) en las Sierras Centrales de Argentina. Latin American Antiquity 23(4): 551-564.

Robledo A., 2016a. Los espacios de combustión en el Alero Deodoro Roca. Arqueología en el Valle de Ongamira, Córdoba. Capítulo 3. Cattáneo G. R. e Izeta A. D. Editores. Arqueología en el valle de Ongamira. Editado por IDACOR CONICET y Museo de Antropología FFyH-UNC

Robledo, A. 2016b. Estudios antracológicos en los espacios de combustión del Alero Deodoro Roca Ongamira (Córdoba). South American Archaeology Series 25, A. D. Izeta (Ed.), Archaeopress Publishing Ltd, Oxford. 169pp.

Serrano, A. 1945. Los Comechingones. Serie Aborígenes Argentinos, Instituto de Arqueología, Lingüística y Folklore de la Universidad Nacional de Córdoba, Córdoba.

Silva, L C. R., M. A. Giorgis, M. Anand, L. Enrico, N. Pérez-Harguindeguy, V. Falczuk, L. L. Tieszen \& M. Cabido. 2011. Evidence of shift in C4 species range in central Argentina during the late Holocene. Plant Soil 349:261-279.

Soibelzon, E., M. Medina \& A. Abba. 2013. Late Holocene Armadillos (Mammalia, Dasypodidae) of the Sierras of Córdoba, Argentina: Zooarchaeology, Diagnostic Characters and their Paleozoological Relevance. Quaternary International 299 p. 72 - 79

Théry-Parisot I., Chabal L. \& Chzavzes J. 2010. Anthracology and taphonomy, from wood gathering to charcoal analysis. A review of the taphonomic processes modifying charcoal assemblages, in archaeological contexts. Paleaogeography, Palaeoclimatology, Palaeoecology 291: 142-153. 
Walker, M. J. C., M. Berkelhammer, S. Björck, L. C. Cwynar, D. A. Fisher, A. J. Long, J. J. Lowe, R. M. Newnham, S. O. Rasmussen\& H. Weiss. 2012. Formal subdivision of the Holocene Series/Epoch: a Discussion Paper by a Working Group of INTIMATE (Integration of ice-core, marine and terrestrial records) and the Subcommission on Quaternary Stratigraphy (International Commission on Stratigraphy). Journal of Quaternary Science 27 (7): 649-659.

Williams, A. N. 2012. The use of summed radiocarbon probability distributions in archaeology: a review of methods. Journal of Archaeological Science 39: 578-589.

Yanes, Y., A. D. Izeta, R. Cattáneo, T. Costa \& S. Gordillo. 2014. Holocene ( 4.5-1.7 cal. kyr BP) paleoenvironmental conditions in central Argentina inferred from entire-shell and intra-shell stable isotope composition of terrestrial gastropods. The Holocene 24 (10): 1193-1205.

Zárate, M. A. 2016. Explorando la historia geológica del alero Deodoro Roca. En: Arqueología en el Valle de Ongamira, Córdoba (2010-2015), R. Cattáneo y A. D. Izeta (Eds). IDACOR-CONICET-UNC, Córdoba, Argentina.

Zuloaga, F. O., O. Morrone, M. J. Belgrano, C. Marticorena \& E. Marchesi. (Eds.) 2008. Catálogo de las Plantas Vasculares del Cono Sur (Argentina, Sur de Brasil, Chile, Paraguay y Uruguay). Monografia Syst. Bot. Missouri Bot. Gard. 107(1): i-xcvi, 1-983; 107(2): i-xx, 985-2286; 107(3): i-xxi, 2287-3348. 